\title{
La Física con una estructurada problematizada: efectos sobre el aprendizaje conceptual, las actitudes e intereses de los estudiantes universitarios \\ (Teaching Physics with a fundamental-problem-based approach: effects on conceptual learning, attitudes and interests of University students)
}

\author{
Carlos Becerra-Labra ${ }^{1}$, Albert Gras-Martí2 y Joaquín Martínez-Torregrosa ${ }^{3}$ \\ ${ }^{1}$ Instituto de Matemática y Física, Universidad de Talca, Chile \\ ${ }^{2}$ Departamento de Física Aplicada, Universidad de Alicante, España \\ ${ }^{3}$ Departamento de Didáctica General y Didácticas Específicas, Universidad de Alicante, España \\ Recebido em 17/5/2006; Aceito em 19/9/2006
}

\begin{abstract}
En el presente artículo se muestra los resultados obtenidos en una investigación realizada para estudiar los efectos que produce una enseñanza de la física con una estructura problematizada en el aprendizaje conceptual, en las actitudes e intereses de los estudiantes. Se da una respuesta fundamentada a las preguntas: una enseñanza de la física a través del planteamiento y resolución de problemas fundamentales, ¿se produce realmente una mejora significativa en el aprendizaje conceptual?, ¿modifica positivamente la actitud e interés de los estudiantes hacia la física? y, ¿se mantiene esta mejora conceptual y actitudinal tiempo después de la instrucción recibida? Palavras-chave: modelo de enseñanza de las ciencias a través de problemas fundamentales, cambio conceptual, cambio actitudinal.
\end{abstract}

We show the results of a research addressing the effects of organizing the teaching of Physics around a problem-based structure. This approach is based upon outlining and addressing the fundamental problems that originated the basic concepts and theoretical framework of the subject matter. We have measured the significant improvement observed in both the students' conceptual understanding and in their attitude and interest towards Physics. We have also investigated whether these learning gains are maintained after the course is over, or a relatively large time has elapsed.

Keywords: science teaching based on fundamental problems, conceptual change, attitudinal change.

\section{Introducción y planteamiento del pro- blema de estudio}

En los últimos años el aumento espectacular de trabajos de investigación en didáctica de las ciencias ha dado como resultado un mayor conocimiento de las dificultades de los estudiantes para aprender ciencias y ha puesto en cuestión, de un modo fundado, el modelo convencional basado fundamentalmente en la creencia de que la transmisión de los conocimientos por el profesor en su estado final (decir lo que es, o explicar cómo se hace, de una manera directa y acabada, junto con la realización de ejercicios) es la única o la mejor forma de lograr que los estudiantes aprendan (paradigma de enseñanza por transmisión verbal) [1-2].

Afortunadamente, la mayoría de estas investigaciones no sólo se han limitado a identificar las deficiencias del modelo transmisión-asimilación de conocimientos ya

\footnotetext{
${ }^{1}$ E-mail: cbecerra@utalca.cl.

Copyright by the Sociedade Brasileira de Física. Printed in Brazil.
}

elaborados (modelo más extendido en Chile), sino que también han generado modelos de enseñanza que pueden competir con éste. Salvando diferencias que podemos considerar menos importantes, la mayoría de dichos modelos consideran que aprender es poder justificar lo que se piensa y que los procesos de producción y aceptación (justificación) de conocimientos que se desarrollan en la vida cotidiana son muy diferentes de los que caracterizan el trabajo científico. Además, dichos modelos afirman que el aprendizaje sólido de los conceptos científicos debe ir acompañado del aprendizaje metodológico, es decir, de formas de producir y aceptar conocimientos que caracterizan el trabajo científico [3-7]. Este desarrollo simultáneo, conceptual-metodológico, se verá favorecido en la medida en que el proceso de enseñanza-aprendizaje se desarrolle en un contexto de (re)construcción de conocimientos (evitando en lo posible, su transmisión en su estado final), en el que existan oportunidades reiteradas y sistemáticas para poner en 
práctica (dentro de lo posible en cada nivel educacional) procesos de justificación típicos de la investigación científica, de la resolución de problemas, y en el que se favorezca la implicación afectiva (actitudinal) necesaria para que esa tarea tan exigente pueda llevarse a cabo.

En efecto, esta idea de que el aprendizaje y la enseñanza de las ciencias se desarrolle como un proceso de (re)construcción de conocimientos en un contexto que se inspire (dentro de lo posible en cada nivel educacional) en la investigación científica es compartida por un amplio abanico de investigadores en didáctica de las ciencias [8-15]. Por su parte, los National Science Education Standards [16] proclaman que "en todos los niveles, la educación científica debe basarse en la metodología de la investigación", como forma de favorecer tanto una actividad significativa, en torno a problemas susceptibles de interesar a los estudiantes, como su progresiva autonomía de juicio y capacidad de participación en tareas colectivas y en decisiones que orientan los desarrollos científico-técnicos.

Por otra parte, en lo que se refiere al proceso de enseñanza-aprendizaje de la física en los primeros niveles universitarios, resulta preocupante el fracaso bastante generalizado de los estudiantes, hecho que se manifiesta en un alto índice de reprobación y abandono. Para producir un cambio significativo de esta situación, hemos desarrollado la enseñanza de la física en el primer curso universitario con una estructura problematizada, donde existan sistemática y reiteradamente oportunidades de poner en práctica las formas de pensamiento y acción de la actividad científica en torno a problemas fundamentales de la física. En otras palabras, queremos probar en qué medida la enseñanza de una asignatura científica universitaria, la mecánica newtoniana en particular, estructurada en forma problematizada, genera un cambio cualitativo y cuantitativo en la enseñanzaaprendizaje de la física. En qué medida se generan oportunidades adecuadas para aprender en el aula, en qué medida mejora el aprendizaje conceptual, las actitudes e intereses de los estudiantes hacia la física.

\section{Fundamentación teórica}

El aprendizaje sólido de los conocimientos científicos implica el desarrollo simultáneo de procesos de producción y aceptación típicos del trabajo científico, y de la implicación axiológica necesaria para que esa tarea tan exigente pueda llevarse a cabo. La planificación de un curso y de los temas en él desarrollados no puede responder simplemente a la lógica que expresa la secuencia: ¿qué objetivos deben lograr los estudiantes?, ¿qué contenidos impartir?, ¿cómo ha de ser el examen para constatar el aprendizaje logrado?

Como hemos señalado, lo que caracteriza el proceso de producción y aceptación de conocimientos científicos es que se desarrolla en un contexto problematizado, donde se abordan situaciones problemáticas de interés y se avanza de una manera tentativa hacia posibles soluciones. Por tanto, la planificación de los temas y de los cursos dentro del modelo de enseñanza de las ciencias como "investigación" dirigida (o por resolución de problemas) obliga a formularse las preguntas que siguen: ¿cómo problematizar el curso y cada uno de los temas incluidos para favorecer el aprendizaje con sentido?, ¿cómo integrar el aprendizaje conceptual, las prácticas de laboratorio y la resolución de problemas de "papel y lápiz" en una estructura problematizada más amplia? Antes de responder a estas preguntas, destacaremos que el presente trabajo de investigación se inserta y se apoya en esfuerzos de innovación e investigación en el campo de la educación científica [17-20].

Para organizar la estructura del curso y de los temas es necesario identificar algunos de los problemas (problemas fundamentales) que están en el origen de las teorías que queremos que pasen a formar parte de los conocimientos de nuestros alumnos, discutir la relevancia de los mismos y planificar una estrategia que permita avanzar en la solución a los problemas planteados, en un ambiente hipotético-deductivo que suministre oportunidades para la apropiación de la epistemología científica. Esto requiere un análisis histórico, epistemológico y didáctico sobre la materia seleccionada para que su estudio sea útil y factible para los alumnos implicados. Este análisis está guiado, por tanto, por preguntas tales como:

¿Qué problemas están en el origen de las teorías que deseamos que pasen a formar parte del bagaje de nuestros alumnos (objetivos/ clave)?

¿Cuáles son/fueron los obstáculos más importantes que hubo que superar para avanzar en la solución a los problemas planteados? ¿Qué ideas, qué razonamientos pueden tener los alumnos sobre los aspectos anteriores que puedan suponer obstáculos para el aprendizaje y que, por tanto, deben ser tomados en consideración? (Identificación de objetivos/ obstáculo).

¿Qué plan concreto de "investigación", secuenciación de actividades, conviene proponer a los estudiantes para avanzar en la solución a los problemas iniciales? (programa-guía de actividades).

Este estudio está dirigido, en definitiva, al diseño de una estructura del curso que permita a los alumnos, con el apoyo, guía y orientación del profesor, enfrentarse a situaciones problemáticas de interés, poniendo en juego buena parte de los procesos de producción y validación de los conocimientos científicos. Más concretamente ello supone:

i. Plantear, en el inicio del curso (y, en su caso, de los grandes bloques o temas que lo compongan) situaciones problemáticas que, inspirándose en las que desde el punto de vista histórico y/o epistemológico, están en el origen de los conocimientos implicados, sirvan de punto de partida para el trabajo de los estudiantes. Por supuesto, debe prestarse atención explícita a que los alumnos se apropien del o los problemas, a que tomen conciencia de su interés, como condición necesaria para su implicación en la tarea.

ii. Diseñar la secuenciación de los temas del curso con una lógica problematizada, es decir, como una posible estrategia para avanzar en la solución a las grandes preguntas iniciales. Esto da lugar a un hilo conductor en el que cada tema se convierte en un problema más 
concreto cuya solución permite avanzar en el problema inicial (problema estructurante), al mismo tiempo que puede generar nuevos problemas, incrementándose así las relaciones entre los distintos temas.

iii. Organizar el índice de cada uno de los temas/problema de forma que responda igualmente a una posible estrategia para avanzar en su solución, es decir, a un plan de "investigación" (programa-guía de actividades) diseñado por el profesor (o, mejor, por equipos de profesores). En este sentido, la estructura o secuencia de apartados del tema debe estar ligada intencional y lógicamente con la problematización inicial. La estructura de los temas no está guiada, por tanto, como es habitual, por los conceptos fundamentales, sino por un intento de plantear y avanzar en problemas fundamentales. De este modo, los conceptos son introducidos funcionalmente como parte del proceso de tratamiento de los problemas planteados y de unificación de campos inicialmente inconexos. Si el conocimiento científico es fruto de un intento de responder preguntas, ¿por qué pretender que los alumnos aprendan respuestas sin conocer las preguntas a las que responden? [21].

iv. En este contexto de resolución de problemas, los conceptos y modelos se introducen, por alumnos y profesor, como tentativas, como hipótesis fundadas, que deben ser puestas a prueba, tanto a través de su capacidad predictiva en situaciones de laboratorio y en el aborde de situaciones problemáticas concretas, que requieren una modelización basada en los mismos (contexto de resolución de problemas, incluyendo la toma de decisiones en situaciones de interés social), como a través del establecimiento de su coherencia con la globalidad de los conocimientos ya establecidos por investigaciones precedentes. La realización de ejercicios, los trabajos prácticos de laboratorio, y la resolución de problemas se integran con sentido, junto a la introducción de conceptos y sus relaciones, dentro de la estructura problematizada [22].

v. Consideramos esencial la realización de recapitulaciones periódicas (recapitulaciones problematizadas) sobre lo que se ha avanzado en la solución al problema planteado, los obstáculos superados y lo que queda por hacer, prestando así especial atención a la regulación y orientación de los alumnos en el desarrollo de la "investigación":

Todo ello constituye una forma de trabajo en el aula que favorece la explicitación de las propias ideas y su confrontación con las de otros, en un ambiente hipotético-deductivo rico en episodios de argumentación y justificación, tan importantes para el aprendizaje de conocimientos científicos [23] y [24]. Se pretende así, en definitiva, crear un ambiente que favorezca simultáneamente la implicación afectiva y la racionalidad científica de todos los implicados (profesor y alumnos) en la resolución de los problemas. Por supuesto, ello exige una cuidadosa planificación de la tarea por parte del profesor, mediante programas de "inves- tigación" (programas de actividades debidamente engarzadas: programa-guía) y exige también dejar tiempo en el aula para que los alumnos piensen, argumenten y refuten.

A continuación, a modo ejemplo se expresa el resultado de este proceso, para el caso específico del curso de mecánica de la partícula. El problema que está en el origen de la mecánica y que sirve de punto de partida para el trabajo de los estudiantes es: encontrar una explicación unitaria, común, al movimiento de todas las cosas (cuerpos celestes y cuerpos terrestres) En otras palabras: ¿existen diferencias esenciales entre los movimientos de todas las cosas, o, podemos encontrar una explicación universal para el movimiento de todas las cosas, independientemente de la naturaleza del objeto que se mueve?

Para que nuestra tarea sea posible y fructífera, es necesario proponer problemas más concretos, fácilmente abordables, que constituyan el hilo conductor del curso y cuyas soluciones nos aproxime a la solución del problema estructurante que se ha planteado. Es decir, para encontrar una explicación universal, única, de por qué las cosas se mueven como lo hacen, es necesario diseñar una estrategia para avanzar en su solución. Un posible plan estratégico podría ser:

i. Tratar de caracterizar los movimientos de los cuerpos ignorando la naturaleza de los mismos. En otras palabras, procuraremos inventar magnitudes físicas para identificar y diferenciar unos movimientos de otros, pero sin tener en cuenta de qué está hecho o cómo es el cuerpo que se mueve; imaginándolo, por ejemplo, como un punto material. (Cinemática de la partícula).

ii. Si logramos describir y diferenciar unos movimientos de otros utilizando los mismos conceptos físicos para todos, será el momento de abordar el siguiente problema: ¿qué es lo que hace que el movimiento de un cuerpo sea de un tipo u otro?, es decir, ¿cómo conseguir que un objeto se mueva como deseamos? (dinámica de la partícula). Dentro del tratamiento de este problema, será de especial interés plantearnos cuestiones como:

a) ¿Qué hace falta para que el movimiento rectilíneo de un objeto sea circular y uniforme como el movimiento de la Luna?

b) Si conseguimos explicar qué ha de ocurrir para que el movimiento de un objeto en la superficie terrestre sea circular y uniforme, ¿qué haría falta para que el movimiento de la Luna se pudiera explicar del mismo modo? (esto justificará la hipótesis de la existencia de la fuerza gravitacional)

c) ¿Qué haría falta para que un objeto terrestre (un coche, una persona) se moviera como la Luna?

d) ¿Podemos explicar la(s) causa(s) de las diferencias entre el movimiento de un globo de hidrógeno y el movimiento de una piedra, cuando ambas se sueltan de la mano?

i. A continuación haremos una recapitulación para ver en qué medida hemos avanzado en la solución de nue- 
stro problema estructurante, es decir, realizaremos una síntesis para ver en qué medida hemos logrado una explicación única, universal, del movimiento de todas las cosas.

ii. Y, finalmente, plantearemos un nuevo problema: ¿existe alguna limitación en la magnitud de los cambios de movimiento que pueden ocurrir en la naturaleza? (Trabajo y energía).

En el anexo (al final del artículo) se representa un es- quema con la estructura problematizada de la mecánica de la partícula. Esta estructura resulta muy útil para la elaboración de recapitulaciones en distintas fases del tema y se concreta en un programa-guía de actividades que son abordadas por los alumnos en el aula (libro de trabajo del alumno). A continuación, en la Tabla 1 se muestra, a modo de ejemplo, dos pequeños fragmentos del programa-guía de actividades.

Tabla 1 - Fragmentos del programa-guía o libro de trabajo del alumno.

Dos fragmentos del inicio programa-guía (libro de trabajo)

(El programa-guía de todo el curso contiene 195 actividades que son abordadas por los alumnos en el aula y/o extra-aula) $(\sim$ A..) son las actividades que realizan los alumnos.

\section{Capítulo 1}

¿Cómo explicar el movimiento de todos los objetos, ignorando la naturaleza del objeto que se mueve?

\section{Planteamiento del problema}

Hay movimiento en todo nuestro alrededor. Lo vemos en las actividades cotidianas de las personas, en los automóviles que pasan por la carretera, en la caída de los cuerpos, en los árboles que se mecen al viento, en la Luna alrededor de la Tierra, etc. En el nivel microscópico también hay movimiento, sólo que no podemos percibirlo directamente: Los electrones que fluyen producen la electricidad, los electrones que vibran generan la luz, etc. El movimiento está en toda la naturaleza, en todo el universo. Es fácil reconocer el movimiento, pero no es tan fácil describirlo.

El tema que vamos a tratar en este curso es el estudio del movimiento de todas las cosas, desde el movimiento de vehículos, como un automóvil o un tren, hasta el movimiento de astros, como la Luna, o simplemente, el movimiento de la caída de un objeto en las proximidades de la superficie terrestre.

Pero antes de empezar esta tarea, es conveniente que nos preguntemos por el interés de la misma:

¿Por qué y para qué vamos a estudiar el movimiento de las cosas?

4. ¿Por qué el movimiento de las cosas ha llamado la atención en todas las épocas, desde la antigüedad hasta nuestros días?

¿Advertimos el fruto de los estudios sobre el movimiento en nuestra actividad cotidiana?

$\sim$ A.1.1 Indica que interés práctico puede tener el estudio del movimiento de: Un vehículo de transporte de personas. Un avión cuando aterriza o despega. Un coche cuando frena. La Luna y el Sol

El interés de estudiar el movimiento de las cosas no sólo se limita a situaciones utilitarias inmediatas, sino que alcanza, como veremos, a la comprensión global del Universo, de la Naturaleza y, por tanto, a la visión que los seres humanos tenemos de nuestra existencia. En efecto, no se trata sólo de medir posiciones o velocidades de los objetos que se mueven, sino que, desde la antigüedad, dichos estudios han ido acompañados de preguntas sobre por qué se mueven los cuerpos o cómo lo hacen; y el interés se ha centrado en las diferencias entre el movimiento de los objetos celestes (como la Luna, los planetas, el Sol u otros) y el movimiento de los objetos terrestres (como la caída de objetos, el movimiento de los proyectiles u otros).

$\sim$ A.1.2 Indica, si las hay, las diferencias entre el movimiento de los astros (como la Luna, los planetas u otros) y el movimiento de los cuerpos en la superficie terrestre (como el movimiento de proyectiles, la caída de los cuerpos u otros).

$\sim$ A.1.3 Si se lanza un objeto, como una piedra, cae al suelo. ¿Por qué no le ocurre lo mismo a la Luna? $\cdots$

Capítulo 2

¿Cómo caracterizar cualquier movimiento y diferenciarlo de otros, ignorando la naturaleza del objeto que se mueve?

\section{Recapitulación}

Siguiendo la estrategia diseñada en el capítulo anterior, el primer problema que vamos a tratar consiste en mostrar que es posible caracterizar cualquier movimiento mediante las mismas magnitudes físicas, independientemente de la naturaleza del objeto que se mueve.

Para darnos cuenta de la complejidad del problema a resolver y para establecer una posible estrategia que nos permita avanzar en su solución, conviene que empecemos por abordar la descripción de un movimiento cualquiera.

$\sim$ A.2.1 Describe con el máximo detalle posible el movimiento de un corredor en una carrera de 100 metros planos, desde que parte hasta que se detiene completamente. Utiliza esquemas, gráficos, inventa valores, etc. Debido a que vamos a construir un conjunto de conocimientos que nos permita caracterizar cualquier movimiento y distinguirlo de otros, conviene que tomemos como referencia vuestras respuestas iniciales a esta cuestión para volver a revisarlas más adelante, y así comprobar en qué medida hemos avanzado en nuestro propósito.

Comenzaremos esta tarea preguntándonos, ¿a qué preguntas tendríamos que dar respuesta si deseamos caracterizar y diferenciar con precisión los movimientos?

$\sim$ A.2.2 Un controlador aéreo sólo puede comunicarse con el piloto de un avión con dificultades mediante la radio. Enumera algunas preguntas que debería hacer al piloto para adquirir una idea precisa del movimiento del avión. De un modo general, ¿qué cuestiones deberíamos tratar para describir con precisión cualquier movimiento? 


\section{Hipótesis y diseños experimentales}

La hipótesis que ha planteado nuestro trabajo de investigación para avanzar en una solución del problema mencionado inicialmente (en el apartado 1), puede formularse de la siguiente manera: "la enseñanza de la física estructurada en forma problematizada produce una mejora significativa en el aprendizaje conceptual y, en las actitudes e intereses de los alumnos". En concreto:

Suministra oportunidades adecuadas en el aula para que los alumnos aprendan con comprensión los conceptos científicos y la significatividad del aprendizaje producido se mantiene tiempo después (varios meses) de la instrucción recibida (se produce el cambio conceptual).

Modifica positivamente la actitud e interés de los estudiantes hacia la física.

Para valorar en qué medida una enseñanza de la física desarrollada con una lógica problematizada suministra oportunidades adecuadas en el aula para que los alumnos del primer curso de física universitaria aprendan con comprensión los conceptos científicos, hemos procedido de la siguiente manera:

a) Confección de un programa-guía o secuencia de actividades con una estructura problematizada (a modo de ejemplo, en la Tabla 1, se incluye dos pequeños fragmentos de éste). Estas actividades han sido desarrolladas en el aula por los alumnos del Grupo Experimental, GE (organizados en pequeños grupos de tres o cuatro alumnos), de tal manera que al resolverlas deben expresar, argumentar y debatir sus ideas, entre ellos y con el profesor. El profesor ha completado (después de cada clase) una red de análisis para expresar su percepción de lo ocurrido durante la sesión y poder mejorar, así, la secuencia de actividades.

b) Se ha diseñado una Prueba Conceptual de diez preguntas (consensuadas entre el profesor del GE y el profesor del GC, Grupo de Control) que ha sido resuelta (en situación de examen) en la penúltima clase del semestre por alumnos de ambos Grupos (GE y GC) A modo de ejemplo, en la Tabla 2, se muestra la pregunta 6 del instrumento diseñado.

c) Para valorar en qué medida se mantiene la significatividad del aprendizaje después (varios meses) de la instrucción recibida, hemos diseñado tres cuestionarios (con preguntas similares a la pregunta planteada en la Tabla 2), que tienen por objetivo medir el nivel de "recuerdo" conceptual en tres momentos posteriores a la finalización del curso. Estos instrumentos se han aplicado, tanto a los alumnos del GE como a los alumnos del GC, a los tres, seis y doce meses después de haber cursado y aprobado la asignatura de física.

Para valorar en qué medida una enseñanza con una estructura problematizada modifica positivamente la actitud e interés de los estudiantes hacia la física, hemos elaborado dos cuestionarios. Las preguntas del cuestionario I se muestran en la Tabla 5 (sólo se ha pasado a los alumnos del GE en la última clase del semestre). Las preguntas del cuestionario II se muestran en la Tabla 6 (se ha pasado a los alumnos que habían aprobado física, tanto del GE y como del GC, doce meses después de haber finalizado el curso).

Tabla 2 - Ejemplo de pregunta de la Prueba Conceptual (contestada en situación de examen).

6. Una persona afirma que es posible conseguir un choque
entre dos bolas de billar idénticas sobre una mesa horizontal
lisa (una de ellas moviéndose con una velocidad $V_{1}$ hacia
la otra que está en reposo) de tal manera que después del
choque la que estaba en reposo se mueva con una veloci-
dad $2 V_{1}$ y la otra bola rebote con una velocidad (- $\left.V_{1}\right)$ (en
sentido opuesto a la dirección inicial), ya que así se cumple
el principio de conservación de la cantidad de movimiento.
Analiza en qué medida puede ser correcta o no dicha afir-
mación, justifica y fundamenta tu respuesta.

\section{Presentación y análisis de resultados}

Conviene destacar que nuestro modelo de enseñanza problematizada (y todo el diseño experimental que acabamos de presentar) se ha aplicado en tres semestres diferentes en el primer nivel de la física universitaria (asignatura de carácter introductoria y semestral, con tres horas-aula semanales). En el presente artículo sólo se muestran los resultados obtenidos en la primera aplicación del modelo, puesto que, lo que es muy importante, los resultados obtenidos en las otras dos aplicaciones han sido prácticamente idénticos.

A los alumnos del primer curso de física universitaria (edad: 17 a 19 años) de la carrera de Agronomía se han dividido (totalmente al azar) en dos secciones diferentes (de 60 alumnos cada una): sección $1=\mathrm{GE}$ (un profesor con una enseñanza problematizada de la física) y sección $2=\mathrm{GC}$ (un profesor con una enseñanza habitual de la física).

Dado la forma de selección de los alumnos y el tamaño de los grupos, para decidir si la diferencia de porcentaje de respuestas correctas es estadísticamente significativa a favor de nuestra hipótesis de trabajo, hemos utilizado el estadístico de prueba z con un nivel de significación del 5\% [25].

El porcentaje de respuestas correctas de la Prueba Conceptual (aplicada en la penúltima clase de física) se muestra en la Tabla 3. Al examen se presentaron 50 alumnos del GC y 57 alumnos del GE, y las diferencias a favor del GE son amplias y estadísticamente significativas en todas las preguntas. A título de ejemplo de las diferencias cualitativas, podemos comentar los resultados de la pregunta 6: la respuesta correcta requiere saber que los cambios que pueden ocurrir en un sistema aislado están restringidos por la conservación de la cantidad de movimiento y la de la energía; y comprobar que, en las condiciones planteadas en la cuestión, aunque se cumple el principio de conservación de la cantidad de movimiento lineal, la energía cinética total 
después de la colisión es 4 veces la energía cinética total antes del choque; por lo que, dicho suceso no es posible. Un $61,4 \%$ de los alumnos del GE da una respuesta correcta frente al 28,0\% del GC y, además, cuando se contabilizan el número de frases con sentido (indicadoras de pensamiento cualitativo y argumentativo), el promedio es mucho mayor en el GE que en el GC.

La Tabla 4 muestra el porcentaje de respuestas correctas de los tres cuestionarios (de las tres pruebas) diseñados para medir el nivel de "recuerdo" conceptual de los alumnos del GE y del GC (sólo aquéllos que aprobaron la asignatura de física) a los tres, seis y doce meses después de la instrucción recibida. En coherencia con los resultados obtenidos de la Prueba Conceptual, los alumnos del GE obtienen un porcentaje de resultados correctos notable y significativamente superior al de los alumnos del GC. El seguimiento realizado muestra claramente que el nivel de "recuerdo" conceptual de los alumnos del GE es significativamente superior al de los alumnos del GC. No sólo esto, los alumnos del Grupo Experimental, en promedio, siguen escribiendo y fundamentando muchos más sus respuestas.

Por tanto, de acuerdo a los resultados obtenidos de la aplicación de la Prueba Conceptual y de los tres cuestionarios de recuerdo (para detectar si se ha producido el cambio conceptual), podemos afirmar que una enseñanza de la física estructurada en forma problematizada suministra oportunidades adecuadas en el aula para que los estudiantes aprendan con comprensión los conceptos científicos y la significatividad del aprendizaje producido se mantiene tiempo después (varios meses) de la instrucción recibida.

Tabla 3 - Resultados de la Prueba Conceptual (en situación de examen).

\begin{tabular}{lcccccccccc}
\hline Respuestas correctas & P 1 & P 2 & P 3 & P 4 & P 5 & P 6 & P 7 & P 8 & P 9 & P 10 \\
\hline GC $(\mathrm{N}=50) \%$ & 32,0 & 40,0 & 30,0 & 28,0 & 34,0 & 28,0 & 28,0 & 26,0 & 32,0 & 20,0 \\
\hline GE $(\mathrm{N}=57) \%$ & 54,4 & 61,4 & 50,9 & 52,6 & 57,9 & 61,4 & 61,4 & 70,2 & 56,1 & 49,1 \\
\hline $\mathrm{P}=$ pregunta & & & & & & & & & &
\end{tabular}

Tabla 4 - Resultados de los cuestionarios para medir el nivel de "recuerdo" conceptual.

\begin{tabular}{lcccccc}
\hline \multirow{2}{*}{ Respuestas correctas } & \multicolumn{2}{c}{3 meses después $\left(1^{\circ}\right.$ Cuest. $)$} & \multicolumn{2}{c}{6 meses después $\left(2^{\circ}\right.$ Cuest. $)$} & \multicolumn{2}{c}{12 meses después $\left(3^{\circ}\right.$ Cuest. $)$} \\
\cline { 2 - 7 } & $\mathrm{GE}(\mathrm{N}=25) \%$ & $\mathrm{GC}(\mathrm{N}=15) \%$ & $\mathrm{GE}(\mathrm{N}=26) \%$ & $\mathrm{GC}(\mathrm{N}=14) \%$ & $\mathrm{GE}(\mathrm{N}=20) \%$ & $\mathrm{GC}(\mathrm{N}=15) \%$ \\
\hline Pregunta 1 & 64,0 & 33,3 & 53,8 & 28,6 & 45,0 & 20,0 \\
Pregunta 2 & 56,0 & 26,7 & 53,8 & 21,4 & 50,0 & 20,0 \\
Pregunta 3 & 68,0 & 20,0 & 57,7 & 21,4 & 45,0 & 13,3 \\
Pregunta 4 & 64,0 & 40,0 & 57,7 & 35,7 & 55,0 & 26,7 \\
Pregunta 5 & 72,0 & 46,7 & 50,0 & 35,7 & 60,0 & 33,3 \\
Pregunta 6 & 60,0 & 26,7 & 53,8 & 28,6 & 55.0 & 26.7 \\
Pregunta 7 & 48,0 & 13,3 & 46,2 & 21,4 & 45,0 & 13,3 \\
\hline
\end{tabular}

Recordemos que la otra consecuencia contrastable de nuestra hipótesis de trabajo es: la metodología propuesta modifica positivamente la actitud e interés de los alumnos hacia la física. En otras palabras, cabe esperar que valoren la metodología propuesta mucho más positivamente que la metodología habitual en los aspectos señalados en el cuestionario I. Dicho cuestionario ha sido contestado por los alumnos del GE, en forma anónima e individual, al finalizar el curso (última clase del semestre).

Para el análisis estadístico de las diferencias de esta valoración comparativa, hemos utilizado el estadístico de prueba t [25] con un nivel de significación del 5\%. El resultado de la valoración comparativa se muestra en la Tabla 5, y se comenta por sí solo. Este resultado muestra profundas diferencias en las "percepciones" que los alumnos tienen entre la metodología propuesta y otras metodologías empleadas en cursos pasados. No sólo han aprendido más de la materia, sino que han "vivido" la enseñanza y el aprendizaje muy positivamente. Tienen la percepción de que esta forma de enseñar les prepara mucho mejor que otras para tareas de alto nivel intelectual, como enfrentarse a problemas nuevos, adquirir hábitos reflexivos superiores, etc., y, lo que es muy esperanzador para los profesores, que ha contribuido a aumentar la actitud positiva e interés hacia la física. Por lo tanto, la diferencia es significativamente superior a favor del modelo propuesto en cada uno de los aspectos valorados en el cuestionario I.

El cuestionario II ha sido contestado, en forma anónima e individual, por los alumnos del GE y del GC doce meses después de la instrucción recibida (se 
ha pasado a los alumnos que han aprobado física y sólo los presentes en la clase de un curso superior al de física). La Tabla 6 (parte1) muestra la respuesta a la pregunta: "¿en cuál o cuáles asignaturas (máximo dos) de los semestres pasados tuviste mayor sensación de aprendizaje? El 65,5\% de los alumnos del GE citan la física entre las dos asignaturas que tuvieron mayor sensación de aprendizaje. En cambio, ningún alumno del GC cita la física dentro de esta categoría, mientras que el 33,3\% la mencionan entre las asignaturas con menor sensación de aprendizaje. Los resultados obtenidos de la valoración de sus respectivas metodologías doce meses después de la instrucción recibida (ver la Tabla 6 , parte 2), muestran claramente que la metodología propuesta sigue siendo percibida por los alumnos del GE de un modo mucho más positiva que otras metodologías. En cambio, los alumnos del GC no perciben de igual forma la metodología usada en su respectivo curso de física.

Los resultados obtenidos en ambos cuestionarios muestran claramente que la valoración de la metodología propuesta es percibida por los alumnos de un modo mucho más positiva que otras metodologías, produciendo un cambio positivo en sus actitudes e intereses. Por tanto, la diferencia es significativa y no hace más que reforzar la validez de nuestra hipótesis de trabajo.

Tabla 5 - Cuestionario I.

\begin{tabular}{|c|c|c|c|}
\hline & \multirow{3}{*}{$\begin{array}{l}\text { Estimado (a) alumno (a), te pedimos que valores de } 0 \text { a } 10 \text { el grado de acuerdo con } \\
\text { las siguientes afirmaciones referidas a la metodología del actual curso de física y la } \\
\text { de los cursos anteriores (10: totalmente de acuerdo; } 5 \text { : indiferente; } 0 \text { : totalmente en } \\
\text { desacuerdo). }\end{array}$} & \multicolumn{2}{|c|}{$\begin{array}{l}\text { Resultado de la valoración } \\
\text { comparativa } \mathrm{GE}(\mathrm{N}=57)\end{array}$} \\
\hline & & $\begin{array}{l}\text { Metodología } \\
\text { actual }\end{array}$ & $\begin{array}{l}\text { Metodología } \\
\text { anterior }\end{array}$ \\
\hline & & $\bar{X}_{M P}(\mathrm{Sd})$ & $\bar{X}_{M A}(\mathrm{Sd})$ \\
\hline 1 & Aumenta la capacidad para resolver todo tipo de problemas y situaciones físicas & $8,0(0,9)$ & $3,7(1,2)$ \\
\hline 2 & $\begin{array}{l}\text { Preparación que produce para saber cómo enfrentarse a problemas y situaciones que } \\
\text { no se han hecho antes }\end{array}$ & $8,1(0,7)$ & $3,5(0,7)$ \\
\hline 3 & Grado en que favorece la comprensión profunda de los conceptos & $8,5(0,6)$ & $3,7(0,9)$ \\
\hline 4 & $\begin{array}{l}\text { Adquisición de hábitos de reflexión, argumentación, planificación de estrategias, y el } \\
\text { análisis de los resultados obtenidos }\end{array}$ & $8,7(0,7)$ & $3,7(0,7)$ \\
\hline 5 & Hace atractiva e interesante la actividad de resolver problemas y situaciones físicas & $8,2(0,8)$ & $3,3(0,9)$ \\
\hline 6 & Contribución a que la física sea más interesante & $8,0(0,9)$ & $3,3(1,0)$ \\
\hline
\end{tabular}

Tabla 6 - Cuestionario II.

\begin{tabular}{|c|c|c|c|c|c|c|}
\hline \multirow{4}{*}{$\begin{array}{l}\text { Parte 1: Estimado(a) alumno(a), estamos haciendo un } \\
\text { análisis para mejorar la enseñanza de la física en nuestra } \\
\text { Universidad, con esta finalidad, te pedimos que respondas a } \\
\text { las siguientes preguntas: }\end{array}$} & \multicolumn{6}{|c|}{ Resultado obtenido doce meses después } \\
\hline & \multicolumn{3}{|c|}{ GE } & \multicolumn{3}{|l|}{$\mathrm{GC}$} \\
\hline & \multicolumn{3}{|l|}{$\mathrm{N}=29$} & \multicolumn{3}{|l|}{$\mathrm{N}=15$} \\
\hline & Asignatura & $\mathrm{n}$ & $\%$ & Asignatura & $\mathrm{n}$ & $\%$ \\
\hline \multirow{2}{*}{$\begin{array}{l}\text { 1. En cuál o cuáles asignaturas (un máximo de dos) de los } \\
\text { semestres pasados tuviste mayor sensación de aprendizaje }\end{array}$} & física & 19 & 65,5 & \multirow{2}{*}{$\begin{array}{l}\text { No aparece } \\
\text { física }\end{array}$} & \multirow[t]{2}{*}{0} & \multirow[t]{2}{*}{0,0} \\
\hline & Bioquímica & 15 & 51,7 & & & \\
\hline \multirow{2}{*}{$\begin{array}{l}\text { 2. En cuál o cuáles asignaturas (un máximo de dos) de los } \\
\text { semestres pasados tuviste menor sensación de aprendizaje }\end{array}$} & \multirow{2}{*}{$\begin{array}{l}\text { No aparece } \\
\text { física }\end{array}$} & \multirow[t]{2}{*}{0} & \multirow[t]{2}{*}{0,0} & física & 5 & 33,3 \\
\hline & & & & Calculo I & 6 & 40,0 \\
\hline $\begin{array}{l}\text { Parte 2: Estimado (a) alumno (a), te pedimos que valores de } \\
0 \text { a } 10 \text { el grado de acuerdo con las siguientes afirmaciones } \\
\text { referidas a la asignatura de física cursada hace algunos meses } \\
\text { (10: totalmente de acuerdo; } 5 \text { : indiferente; } 0 \text { : totalmente en } \\
\text { desacuerdo). }\end{array}$ & \multicolumn{3}{|c|}{ GE N $=29$ Media $(\mathrm{Sd})$} & \multicolumn{3}{|c|}{ GC N $=15$ Media (Sd) } \\
\hline 1. Contribuyó a mejorar mis actitudes hacia la física & \multicolumn{3}{|l|}{$8,5(0,8)$} & \multicolumn{3}{|l|}{$3,2(0,9)$} \\
\hline $\begin{array}{l}\text { 2. Lo importante para aprobar era comprender verdad, no } \\
\text { memorizar fórmulas }\end{array}$ & \multicolumn{3}{|l|}{$9,0(0,5)$} & \multicolumn{3}{|l|}{$6,5(1,3)$} \\
\hline $\begin{array}{l}\text { 3. La forma de desarrollar la clase favorecía especialmente el } \\
\text { aprender "de verdad" }\end{array}$ & \multicolumn{3}{|l|}{$8,7(0,7)$} & \multicolumn{3}{|l|}{$3,5(0,8)$} \\
\hline $\begin{array}{l}\text { 4. Contribuyó a mejorar mi capacidad para pensar, para en- } \\
\text { frentar situaciones novedosas }\end{array}$ & \multicolumn{3}{|l|}{$8,6(0,6)$} & \multicolumn{3}{|l|}{$4,1(1,1)$} \\
\hline $\begin{array}{l}\text { 5. Me resultó difícil: no había relación entre lo que se hacía } \\
\text { en clase y lo que se exigía en el examen }\end{array}$ & \multicolumn{3}{|l|}{$0,0(-)$} & \multicolumn{3}{|l|}{$8,2(1,0)$} \\
\hline $\begin{array}{l}\text { 6. Si tuviera otra asignatura de física me gustaría que se } \\
\text { enseñara del mismo modo }\end{array}$ & \multicolumn{3}{|l|}{$8,4(1,2)$} & \multicolumn{3}{|l|}{$2,5(1,2)$} \\
\hline $\begin{array}{l}\text { 7. A lo largo de la asignatura estuve orientado: sabía por qué } \\
\text { y para qué hacíamos las cosas (frente a estar haciendo cosas } \\
\text { sin saber el interés y la finalidad de las mismas) }\end{array}$ & \multicolumn{3}{|l|}{$8,3(1,1)$} & \multicolumn{3}{|l|}{$3,5(1,3)$} \\
\hline
\end{tabular}




\section{Conclusiones y perspectivas}

Tras los resultados obtenidos de nuestro trabajo de investigación (que, recordamos, han sido reproducidos durante tres semestres, aunque aquí sólo se han mostrado los del primero), podemos afirmar que una enseñanza de la física, en el primer curso universitario, con una estructura problematizada produce una mejora significativa en el aprendizaje y en el rendimiento académico de la mayoría de los alumnos y no sólo para una elite. En otras palabras, se ha probado que una enseñanza de la física organizada en torno al planteamiento y solución de problemas fundamentales produce una mejora significativa en el aprendizaje conceptual $\mathrm{y}$, en las actitudes e intereses de los estudiantes hacia la física. Además, abre nuevos y fructíferos modos de actuación en las aulas de los primeros niveles universitarios, a través de la implicación de profesores y alumnos en una tarea de carácter colectivo, en sintonía con las pautas del trabajo científico.

Aunque en este artículo nos hemos limitado al aprendizaje conceptual y a las actitudes generadas, pronto someteremos a consideración de la comunidad científica nuestro estudio sobre los efectos que produce una enseñanza problematizada de la física en la resolución de problemas de "lápiz y papel" [15]: ¿se produce realmente en los alumnos un aumento significativo en la capacidad para resolver problemas de "lápiz y papel"?, ¿cómo se aplica nuestro modelo de enseñanza problematizada en la resolución de problemas de "lápiz y papel"?

\section{Anexo}

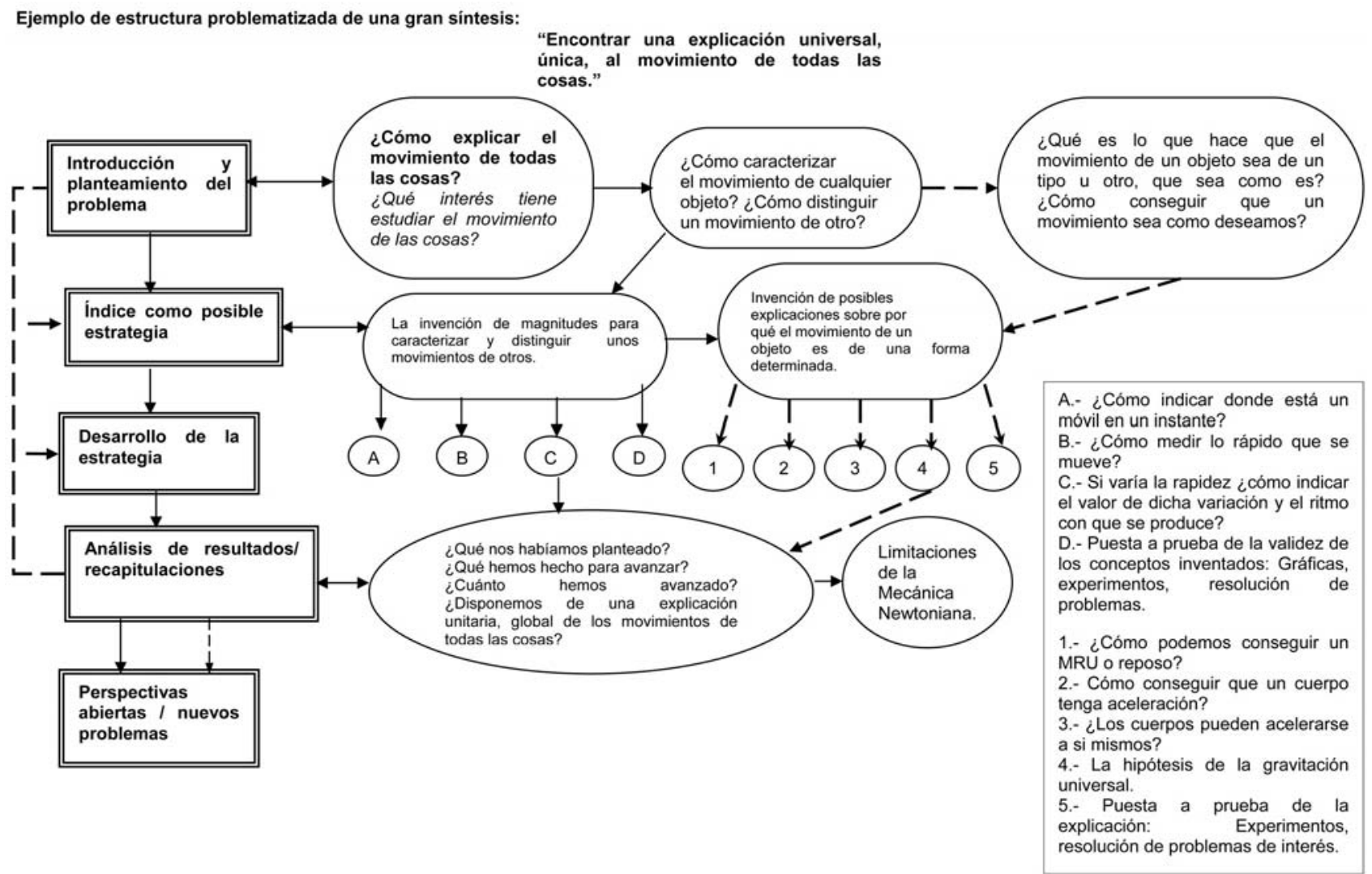

\section{Referencias}

[1] J.D. Novak, Teoría y Práctica de la Educación (Alianza, Madrid, 1982).

[2] D. Gil, Enseñanza de las Ciencias 1, 26 (1983).

[3] D. Hodson, International Journal of Science Education 14, 541 (1992).

[4] R. Duschl y D. Gitomer, Journal for Research in Science Teaching 28, 839 (1991).
[5] A. Sardá y N. Sanmartí, Enseñanza de las Ciencias 18, 405 (2000).

[6] M.P. Jiménez Aleixandre, in Ponencia en el VI Congreso de Enseñanza de las Ciencias, Barcelona, 2001.

[7] M.P. Jiménez Aleixandre y J. Díaz de Bustamante, Enseñanza de las Ciencias 21, 359 (2003).

[8] R. Osborne y M. Wittrok, Studies in Science Education 12, 59 (1985) 
[9] R. Driver y V. Oldham, Studies in Science Education 13, 105 (1986).

[10] J.F. Osborne, Science Education 80, 53 (1996).

[11] D. Gil y J. Martínez-Torregrosa, Investigación en la Escuela 33 (1978).

[12] L. Bencze y D. Hodson, Journal of Research in Science Teaching 36, 521 (1999).

[13] U. Zoller, Journal of Research in Science Teaching 36, 583 (1999).

[14] J. Guisasola, C. Furió, M. Ceberio y J.L. Zubimend, Enseñanza de las Ciencias extra, 17 (2003).

[15] C. Becerra-Labra, A. Gras-Martí y J. MartínezTorregrosa, Rev. Bras. Ens. Fís. 27, 299 (2005).

[16] National Science Education Standards, National Committee on Science Education Standards and Assessment, National Research Council, 1996.

[17] P. Black, in Physics 2000: Physics as it Enters a New Millenium, IUPAP (International Union of Pure and Applied Physics), Paul Black, Gordon Drake, and Leonard Jossem, eds. (2000) (http://www.physics.ohiostate.edu/ jossem/IUPAP/P2000.pdf).
[18] D. Gil y A. Vilches, Revista Española de Física 13, 10 (1999).

[19] C. Monereo y J.I. Pozo (eds), La Universidad ante la Nueva Cultura Educativa (Madrid, Síntesis, 2003).

[20] D. Gil-Pérez, J. Guisasola, A. Moreno, A. Cachapuz, A. M. Pessoa de Carvalho, J. Martínez-Torregrosa, J. Salinas, P. Valdés, E. González, A. Gené Duch, A. DumasCarré, H. Tricárico y R. Gallego, Science \& Education 11, 557 (2002).

[21] J. Otero, European Journal of Science Education 7, 361 (1985).

[22] D. Gil, C. Furió, P. Valdés, J. Salinas, J. MartínezTorregrosa, J. Guisáosla, E. González, A. DumasCarré, M. Goffard y A. Pessoa de Carvalho, Enseñanza de las Ciencias 17, 311 (1999).

[23] R. Driver y V. Oldham, Studies in Science Education 13, 105 (1986).

[24] R. Driver, P. Newton y J. Osborne, Science Education 84, 287 (2000).

[25] J. Welkowitz, R.B. Ewen y J. Cohen, Estadística Aplicada a las Ciencias de la Educación (Santillana S.A., Madrid, 1981). 